\title{
Omnidirectional broadband insulating device for flexural waves in thin plates
}

\author{
Alfonso Climente, Daniel Torrent, and José Sánchez-Dehesa ${ }^{\text {a) }}$ \\ Wave Phenomena Group, Universitat Politècnica de València, C/Camino de vera s.n. (Edificio 7F), \\ ES-46022 Valencia, Spain
}

(Received 7 October 2013; accepted 17 November 2013; published online 4 December 2013)

\begin{abstract}
This work presents a gradient index device for insulating from vibrations a circular area of a thin plate. The gradient of the refractive index is achieved exploiting the thickness-dependence of the dispersion relation of flexural waves in thin plates. A well-like thickness profile in an annular region of the plate is used to mimic the combination of an attractive and repulsive potentials, focusing waves at its bottom and dissipating them by means of an absorptive layer placed on top. The central area is therefore isolated from vibrations, while they are dissipated at the bottom of the well. Simulations have been done using the multilayer multiple scattering method and the results prove their broadband efficiency and omnidirectional properties. (C) 2013 AIP Publishing LLC. [http://dx.doi.org/10.1063/1.4839375]
\end{abstract}

\section{INTRODUCTION}

The study of the propagation of flexural waves in thin plates has received an increasing attention recently, due to the possibilities in their control offered by new structures like phononic crystals, ${ }^{1-6}$ arrays of attached resonators, ${ }^{7-9}$ or transformation-coordinate based devices. ${ }^{10,11}$ Therefore, applications such as cloaking shells ${ }^{12}$ or negative refractive lenses ${ }^{4,13}$ have been experimentally demonstrated, opening the door to new and exciting devices for the control of vibrations.

In addition to all these phenomena related with classical waves in general, the propagation of flexural waves in thin plates presents the peculiarity that it can be controlled by means of thickness variations, as was theoretically studied by Krylov. ${ }^{14}$ Known as wedges, these structures produce a gradual reduction in the velocity of the wave by changing the plate's local thickness. Experimental investigations have been carried out on a variety of plate-like and beam-like structures. $^{15,16}$

The principle of wave control by locally changing the plate's thickness has been applied to achieve efficient damping of flexural waves in plate-like structures using the so called "acoustic black holes" $(\mathrm{ABH}),{ }^{17}$ which consist of specifically designed pits with small pieces of absorbing materials attached in the middle. ${ }^{18}$ Recently, O'Boy and Krylov ${ }^{19}$ introduced a modification in the $\mathrm{ABH}$ by perforating a hole in the center of the pit, and Bowyer and coworkers ${ }^{20}$ studied the effect of imperfections in the fabrication method of the $\mathrm{ABH}$ and the result of placing multiple $\mathrm{ABH}$ together in a plate. ${ }^{21}$ For more information on these topics, see Ref. 22.

In this work, a device for isolating a given area in a thin plate from vibrations is designed and numerically tested. This objective is accomplished by surrounding it by a properly designed thickness-inhomogeneous region, which will attract and dissipate vibrations on the plate, accomplishing then a double objective. From one side, the central region

\footnotetext{
a) Author to whom correspondence should be addressed. Electronic mail: jsdehesa@upvnet.upv.es
}

will be properly isolated, and from the other one, the device will dissipate the vibrations on the plate. Our work is based on the work introduced by Krylov et al. ${ }^{14-17}$ and the RossUngar-Kerwin (RKU) model. ${ }^{18}$

The paper is organized as follows: Section II introduces the Kirchoff-Love approximation for modeling the behavior of flexural waves in thin plates and the method to design the structure is explained. Section III explains the RKU theory used to model the behavior of an absorbing layer placed on top of an elastic plate. In Sec. IV, the design and optimization of the device are explained and in Sec. V the performance of the device is tested using a numerical simulator based on a multiple scattering algorithm. Finally, the conclusions are presented in the last section and the numerical algorithm is explained in the Appendix.

\section{THEORY}

The equation of motion describing flexural waves in thin plates is modeled using the Kirchoff-Love approximation ${ }^{23-25}$ in which the vertical displacement $W(x, y)$ of the plate is obtained from the bi-Helmholtz equation (assuming harmonic time dependence of frequency $\omega$ )

$$
D \nabla^{4} W(x, y)-\rho h \omega^{2} W(x, y)=0,
$$

where $D=E h^{3} / 12\left(1-\nu^{2}\right)$ is the flexural rigidity, $\rho$ the mass density, $h$ the thickness of the plate, $E$ the Young Modulus, and $\nu$ the Poisson ratio. For plane wave propagation with wavenumber $k$, the above equation gives a quadratic dispersion relation

$$
k^{4}=\frac{\rho h \omega^{2}}{D},
$$

and a phase velocity

$$
c^{4}=\left(\frac{\omega}{k}\right)^{4}=\omega^{2} \frac{E h^{3}}{12\left(1-\nu^{2}\right) \rho h} .
$$


It can be seen that the phase velocity $c$ is a function not only of the physical properties of the plate, but it also depends on its thickness. This dependence allows the design of gradient index devices for flexural waves easily by means of local variation of the plate's thickness. Thus, the refractive index as a function of the plate's thickness is (in polar coordinates)

$$
n(r, \theta)=\frac{c_{b}}{c(r, \theta)}=\sqrt{\frac{h_{b}}{h(r, \theta)}},
$$

$c_{b}$ being the wave speed in the background, $h_{b}$ the background's thickness, and $h(r, \theta)$ the position-dependent thickness. It has been assumed that all the other elastic properties of the plate remain unchanged. Equation (4) describes how the refractive index increases with decreasing thickness. It is important to notice that, despite of being an intrinsically dispersive medium, the refractive index does not depend on the frequency of the wave; thus, the functionality of the designed devices will be limited by the accuracy of the flexural wave model only.

Figure 1 shows a schematic view of the gradient index device analyzed in this work. It consists of a circularly symmetric region in which the thickness of the plate is gradually changed according to the desired functionality. In this case, a well-like profile is drilled surrounding a central area, which is the region to be isolated from external vibrations. Following the analogy with acoustic and electromagnetic waves for similar devices, ${ }^{26,27}$ the objective of the decreasing height (increasing refractive index) region is to act as an "attractive" potential, so that it tends to concentrate vibrations, while the inner region of increasing height (decreasing refractive index) will act as a "repulsive" potential, isolating in this way the central region. All waves traveling around this device will be concentrated at the bottom of the well where they will be dissipated.

The proposed structure is theoretically studied by means of a multiple scattering method, where the variation of the height is discretized and then the structure is modeled as a multilayered shell. This method requires the application of the proper boundary conditions at each layer, for which it is necessary to know their physical properties. Note that the only parameter that changes from layer to layer is the plate's thickness, except at the bottom of the well, where a dissipative material is placed. Section III describes the model employed in analyzing the absorbing layer.

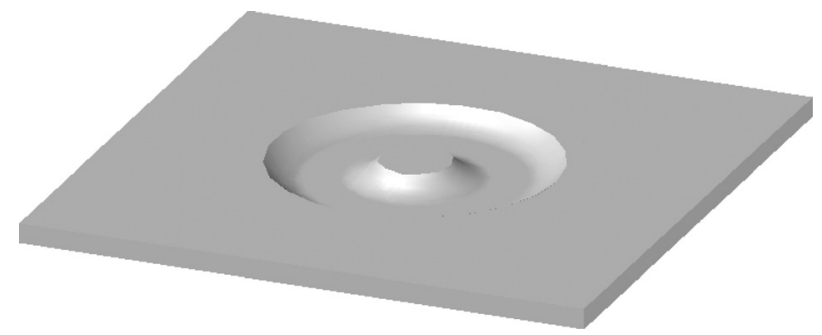

FIG. 1. Schematic view of the structure studied in the present work. The central circular region is surrounded by a thickness-varying shell so that it is isolated from the propagation of flexural waves on the plate.

\section{ABSORBING LAYER MODEL}

The goal of the proposed device is to concentrate waves in a given region where they are dissipates. This dissipation is made by placing an absorptive material of thickness $\delta$ in contact with the plate of thickness $h_{a}$ in the region of interest, as shown in Fig. 2. To properly apply the multiple scattering method described in the Appendix, this region must be modeled as a single layer with a given Young modulus $E_{c}$, thickness $h_{c}$, Poisson ration $\nu_{c}$, and density $\rho_{c}$. Absorption is introduced in the model by adding a complex part in the Young modulus called the loss factor $\eta$, such that $\hat{E}=E(1+i \eta)$; therefore, the model must also provide the composite loss factor $\eta_{c}$.

Using the RKU model, ${ }^{18}$ it is possible to describe the system plate-absorptive material as a single composite. This model states that the wavenumber of the composite material is

$$
k_{c}^{4}=\frac{12 \omega^{2} \rho_{a}\left(1-\nu_{a}^{2}\right)}{E_{a} h_{a}^{2}}\left[\frac{1+\rho_{r} h_{r}}{\left(1-i \eta_{a}\right)+\left(1-i \eta_{\ell}\right) h_{r} E_{r} \alpha}\right],
$$

where the subindices $a$ and $\ell$ stands for the parameters of the plate and absorbing layer, respectively, and $\rho_{r}=\rho_{\ell} / \rho_{a}$, $h_{r}=\delta / h_{a}, E_{r}=E_{\ell} / E_{a}$, and $\alpha=3+6 h_{r}+4 h_{r}^{2}$.

The thickness of the composite is simply the total thickness of the two layers

$$
h_{c}=h_{a}+\delta,
$$

while its density is the volume average of the densities of the two materials

$$
\rho_{c}=\frac{\rho_{a} h_{a}+\rho_{\ell} \delta}{h_{c}} .
$$

It is assumed that the Poisson ratio of the composite is the same as that of the plate

$$
\nu_{c}=\nu_{a} .
$$

From Eqs. (5)-(8) and (2), the flexural rigidity is obtained as

$$
\begin{aligned}
D_{c}= & \frac{\omega^{2} \rho_{c} h_{c}}{k_{c}^{4}}=\frac{h_{a}^{3}}{12\left(1-\nu_{a}^{2}\right)} \\
& \times E_{a}\left[\left(1+h_{r} E_{r} \alpha\right)-i\left(\eta_{a}+\eta_{\ell} h_{r} E_{r} \alpha\right)\right] .
\end{aligned}
$$

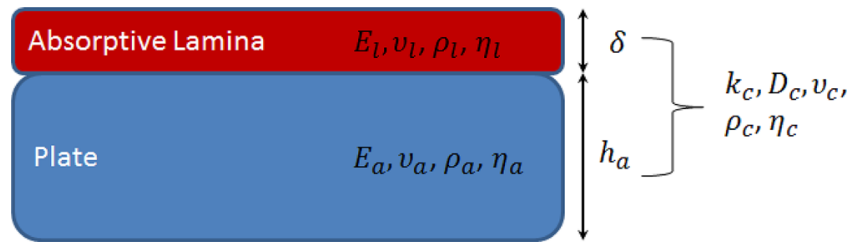

FIG. 2. Geometry employed to dissipate the vibrations on the plate. An absorbing layer (thickness $\delta$ ) is placed on the top of the plate (thickness $h_{a}$ ). Each layer has its own elastic parameters that combine to produce a composite material (thickness $h_{a}$ ), with new elastic parameters. This effective material is the one considered in the model. 
TABLE I. Elastic parameters of the materials used in this work. ${ }^{28}$

\begin{tabular}{lcc}
\hline \hline & Aluminum & Moulded Polystyrene \\
\hline Young modulus $E$ & $78.97 \mathrm{Gpa}$ & $7.8 \mathrm{Gpa}$ \\
Mass density $\rho$ & $2700 \mathrm{~kg} / \mathrm{m}^{3}$ & $1400 \mathrm{~kg} / \mathrm{m}^{3}$ \\
Poisson ratio $\nu$ & 0.33 & 0.34 \\
Loss factor $\eta$ & 0.0001 & 0.1 \\
\hline \hline
\end{tabular}

Finally, knowing that $E_{c}=D_{c} 12\left(1-\nu_{c}^{2}\right) / h_{c}^{3}$, the Young modulus of the composite is obtained and its loss factor is found as

$$
\eta_{c}=\frac{\Im\left\{E_{c}\right\}}{\Re\left\{E_{c}\right\}}=\frac{\eta_{a}+\eta_{\ell} h_{r} E_{r} \alpha}{1+h_{r} E_{r} \alpha} .
$$

In this work, an aluminum plate and a moulded Polystyrene layer has been used. Table I reports the materials' properties. ${ }^{28}$ Figure 3 shows how the variation of the composite loss factor with the relative Young modulus $E_{r}=E_{\ell} / E_{a}$ for different values of $\eta_{\ell}$. The thickness of the aluminum plate is $h_{a}=0.5 \mathrm{~mm}$, and the thickness of the layer is $\delta=0.5 \mathrm{~mm}$. It is observed that $\eta_{c}$ approaches the loss factor of the absorptive layer $\eta_{\ell}$ as the normalized Young Modulus $E_{r} \gg 0.5$, as expected from Eq. (10).

Figure 4 shows how the loss factor changes with the thickness of the plate and the layer. The material properties are given in Table I. Note that when the thickness of the plate decreases, a minor change in the thickness of the layer changes the loss factor greatly. Given that the thickness of both the absorptive layer and the plate can be tailored, their values are optimized in order to maximize the device's efficiency. The optimization method is explained in Sec. IV.

\section{DESIGN AND OPTIMIZATION}

The full studied device consists of five regions as shown in Figure 5 (upper panel), where each region is drawn in a

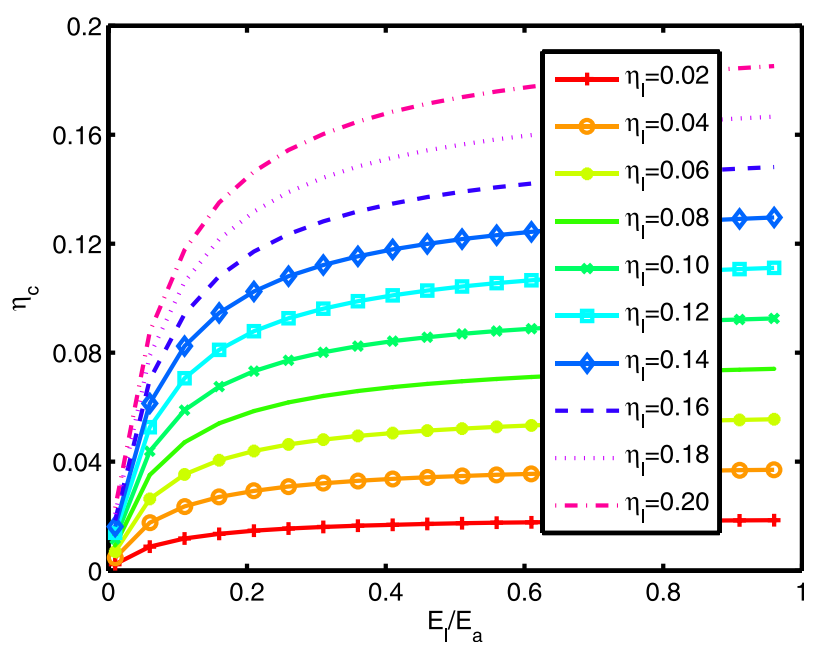

FIG. 3. Variation of the composite loss factor $\eta_{c}$ with the normalized Young Modulus $E_{r}=E_{\ell} / E_{a}$, for different values of the loss factor of the absorptive layer $\eta_{\ell}$ and for $h_{a}=0.5 \mathrm{~mm}$ and $\delta=0.5 \mathrm{~mm}$.

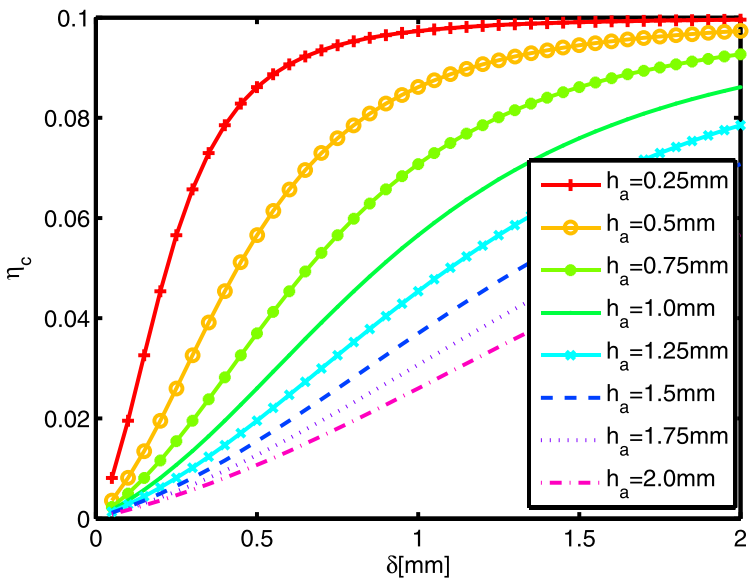

FIG. 4. Variation of the composite loss factor $\eta_{c}$ with the thickness of the layer $\delta$ for different plate thickness $h_{a}$.

different color. Figure 5 (lower panel) shows the variation of the thickness of the plate according to the following function:

$h(r)=\left\{\begin{array}{lc}h=h_{b} & r \leq R_{c} \\ h=\frac{h_{b}-h_{\text {min }}}{\left(R_{c}-R_{r p}\right)^{2}}\left(r-R_{r p}\right)^{2}+h_{\text {min }} & R_{c}<r \leq R_{r p} \\ h=h_{\text {min }} & R_{r p}<r \leq R_{a b} \\ h=\frac{h_{b}-h_{\text {min }}}{\left(R_{a p}-R_{a b}\right)^{2}}\left(r-R_{a b}\right)^{2}+h_{\text {min }} & R_{a b}<r \leq R_{a p} \\ h=h_{b} & R_{a p}<r,\end{array}\right.$

where $h_{b}=10 \mathrm{~mm}$ is the thickness of the plate in the background, $h_{\text {min }}=0.5 \mathrm{~mm}$ is the minimum thickness of the plate (prior to the optimization process), $R_{c}=15 \mathrm{~cm}$ is the radius of the core, $R_{r p}=30 \mathrm{~cm}$ is the radius of the "repulsive potential" shell, $R_{a b}=45 \mathrm{~cm}$ is the radius of the absorptive shell, and $R_{a p}=60 \mathrm{~cm}$ is the radius of the "attractive potential" shell.

To maximize the energy transfer through all the layers, the system requires a good matching between each interface, so that the reflections of incoming waves be minimum. Boundary conditions of the Kirchoff-Love approximation are continuity of the displacement $W$, its radial derivative $\partial_{r} W$, the conservation of the bending moment and the generalized Kirchoff stress, as given by Eqs. (A12) in the Appendix. These equations are functions of the flexural rigidity $D(r)$ and the wavenumber $k(r)$ ( $\nu$ does not change), so that, in order to minimize reflections when changing from one region to the other one, these values have to be continuous.

Although the thickness values given from Eq. (11) provide this continuity, once the effect of the absorbing layer is added, a mismatch between the layers surrounding the absorptive shell occurs. An optimization process solves this problem. Two parameters have been optimized, the thickness of the absorbing layer $(\delta)$ and the thickness of the absorbing plate $\left(h_{a} \leq h_{\text {min }}\right)$. Figure 5 (lower panel) shows, in the absorption region, the original value $h_{\text {min }}$ (dashed line), the new thickness value $h_{a}<h_{\text {min }}$ (continuous line) and, finally, 


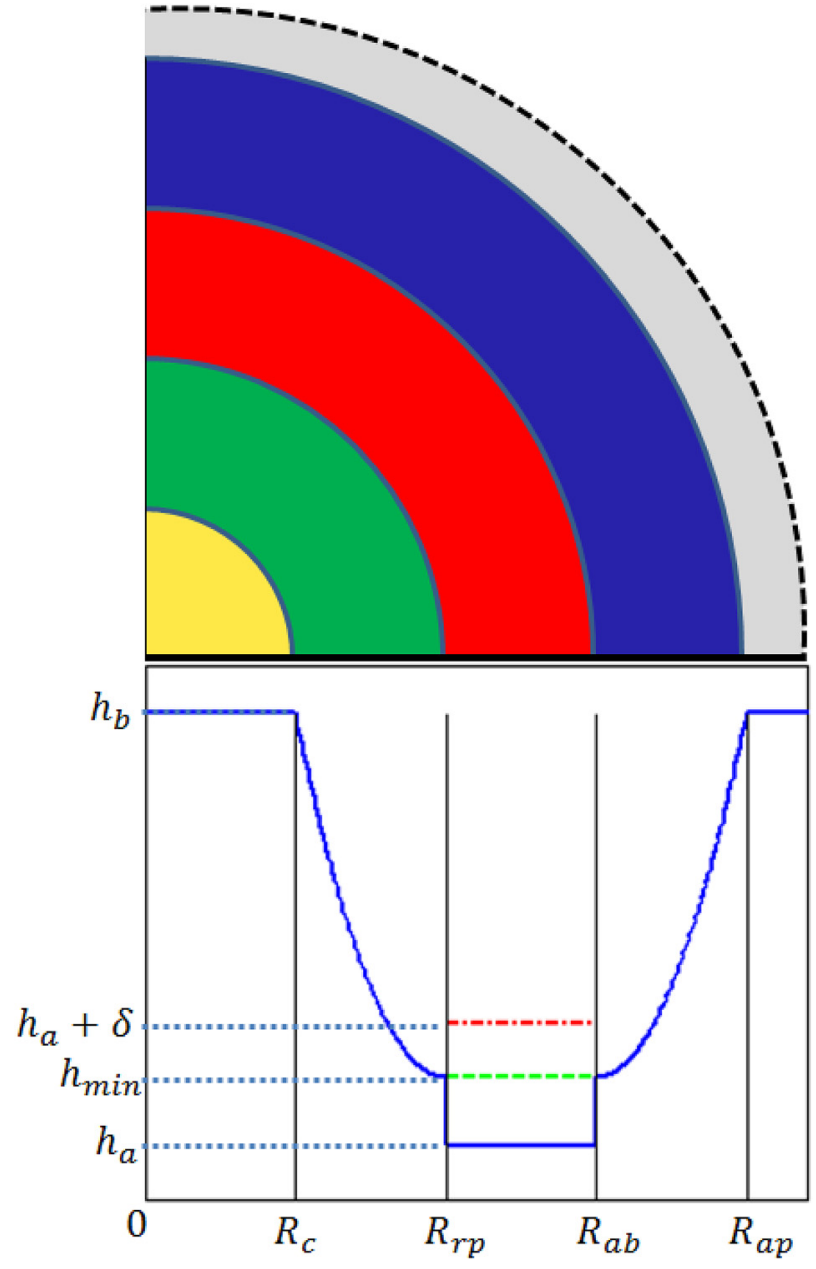

FIG. 5. Different regions defined in the gradient index device (upper panel) and variation of the thickness of the plate (lower panel). The core is defined by $r<R_{c}$ (yellow) and corresponds to the area to be isolated from vibrations. Region $R_{c}<r \leq R_{r p}$ is the repulsive potential shell (green), region $R_{r p}<$ $r \leq R_{a b}$ is the absorbing shell (red), and region $R_{a b}<r \leq R_{r p}$ is the attractive potential shell (blue). The grey region corresponds to the background and it extents towards infinity.

and the total thickness after adding the absorbing layer $h_{a}+\delta$ (dashed-dotted line). The goal is to obtain a relative error lower than $0.1 \%$ for the flexural rigidity and the wavenumber, i.e.,

$$
\begin{aligned}
\epsilon_{D(r)} & =\left|D(r)_{\text {target }}-D(r)\right| / D(r)_{\text {target }}, \\
\epsilon_{k(r)} & =\left|k(r)_{\text {target }}-k(r)\right| / k(r)_{\text {target }},
\end{aligned}
$$

of less than $0.1 \%$. After the optimization, the values for the new thickness of the plate and the layer are $h_{a}=0.44 h_{\text {min }}$ and $\delta=0.55 \mathrm{~mm}$, respectively. Also, the composite loss factor achieved was $\eta_{c} \approx 0.1$.

\section{RESULTS AND DISCUSSION}

Using the design described in Sec. IV and the numerical algorithm described in the Appendix, simulations have been done to test the performance of the device. Each region has

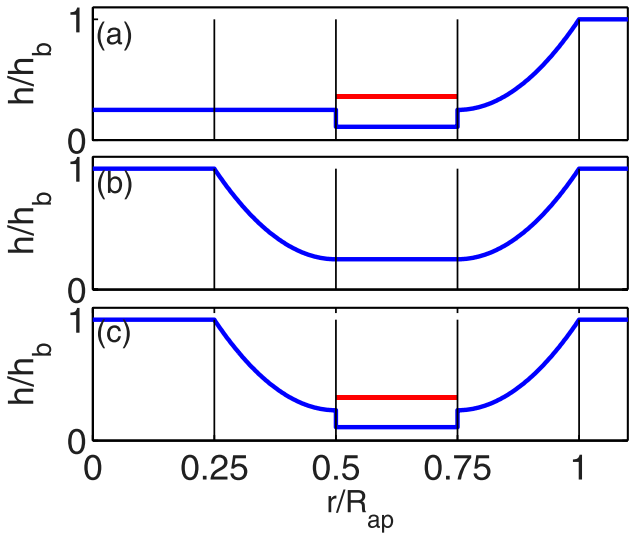

FIG. 6. Thickness variation as a function of the distance $r$ for the three structures studied in this work. The region wanted to be free of vibration is $r \leq R_{a p} / 4$. (a) Attractive potential with the absorptive layer. (b) Attractive and repulsive potentials without the absorptive layer. (c) Full isolating device.

been discretized into $N=100$ layers, which has been shown to be a good approximation to the ideal continuous device for the wavelengths of interest.

For comparison purposes, the three configurations shown in Fig. 6 have been studied. The first one is a device with the same geometrical characteristics as the designed one, but without the repulsive potential (Fig. 6(a)); the second one is a device with the same geometrical characteristics as the designed one, but without the absorptive layer and the optimization (Fig. 6(b)); and the third one is the designed device (Fig. 6(c)).

To further understand what is occurring inside the three configurations, let us consider the displacement field illustrated in Fig. 7. It shows the modulus of the displacement in the $z$-direction when a plane wave with wavenumbers $k R_{a p}=15$ (left panels) and $k R_{a p}=35$ (right panels), impinges the devices under study. The white circles represent the boundaries defined by Eq. (11). Panels (a) represent the plot of the device without the repulsive potential. Notice that, although the system presents absorption, the wave is focused into the core and a high amplitude is achieved. Panels (b) are for the device without the absorptive layer, but adding the repulsive potential shell. It is observed that the wave is expelled from the core and the amplitude decreases in comparison to the previous panels, even though it does not have absorption. Finally, panels (c) correspond to the designed optimum device. By introducing the absorptive layer, the wave amplitude in the core is further reduced. Notice that the panel in the second row has the same pattern as the one in the third row but without the attenuation.

For comparison purposes, we have introduced the vibration average in the region $i$ defined as

$$
\left\langle|W|^{2}\right\rangle_{i}=\frac{1}{S_{i}} \iint_{S_{i}}|W(r, \theta)|^{2} d S,
$$

where $S_{i}$ is the area defined by $R_{i}<r<R_{i+1}$, with $R_{i} \in\left[\infty, R_{a p}, R_{a b}, R_{r p}, R_{c}, 0\right]$.

Figure 8, panels a-d, shows the vibration average produced in the four regions defining the device: the attractive 

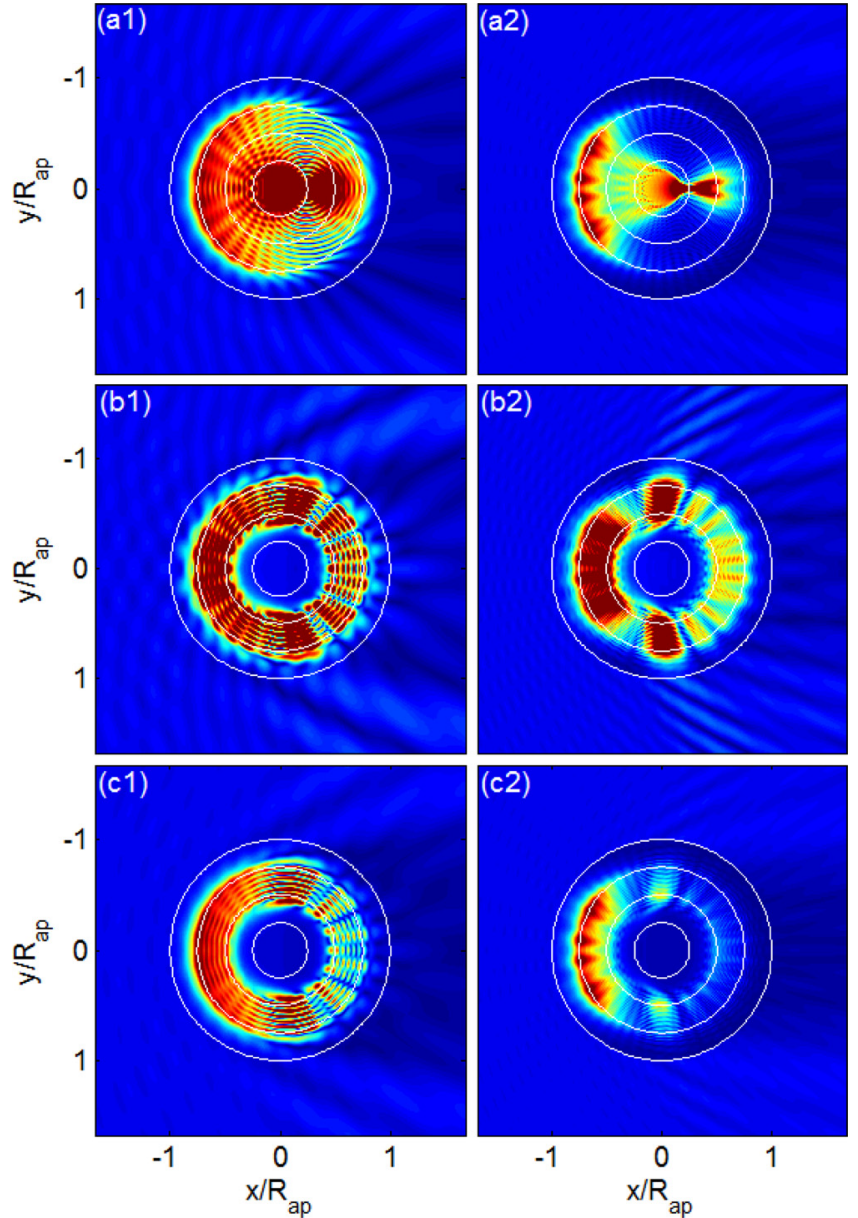

FIG. 7. Modulus of the displacement in the $z$-direction when a plane wave with wavenumber $k R_{a p}=15$ (left panels) and $k R_{a p}=35$ (right panels) impinges on the three devices tested in this work. Displacement produced by the device without the repulsive potential (a), by the device without absorption (b), and by the designed device (c).

potential shell, the absorbing shell, the repulsive shell, and the core, respectively. Each figure shows the values of the vibration average for the designed device (continuous line), the device without absorption shell (dashed line), and the device without the repulsive potential shell (dotted line). Notice that in general, the vibration average decreases with increasing frequency, due to the frequency response of the absorptive layer. Some peaks are observed in the device without the absorption shell due to resonances of the structure, which disappear once dissipation is introduced in the device. Overall, the designed device has the minimum vibration average in all the regions, which shows its efficiency not only for dissipating vibration energy but also for isolating a given region from these vibrations.

\section{SUMMARY}

An omnidirectional and broadband device allowing the isolation of a given circular region from the flexural waves propagating in a thin plate has been theoretically demonstrated. It is based on a circular gradient index lens that guide flexural waves to an annular region where the vibrations are dissipated. The dissipation in the annular region has been modeled using the RKU approach by considering an
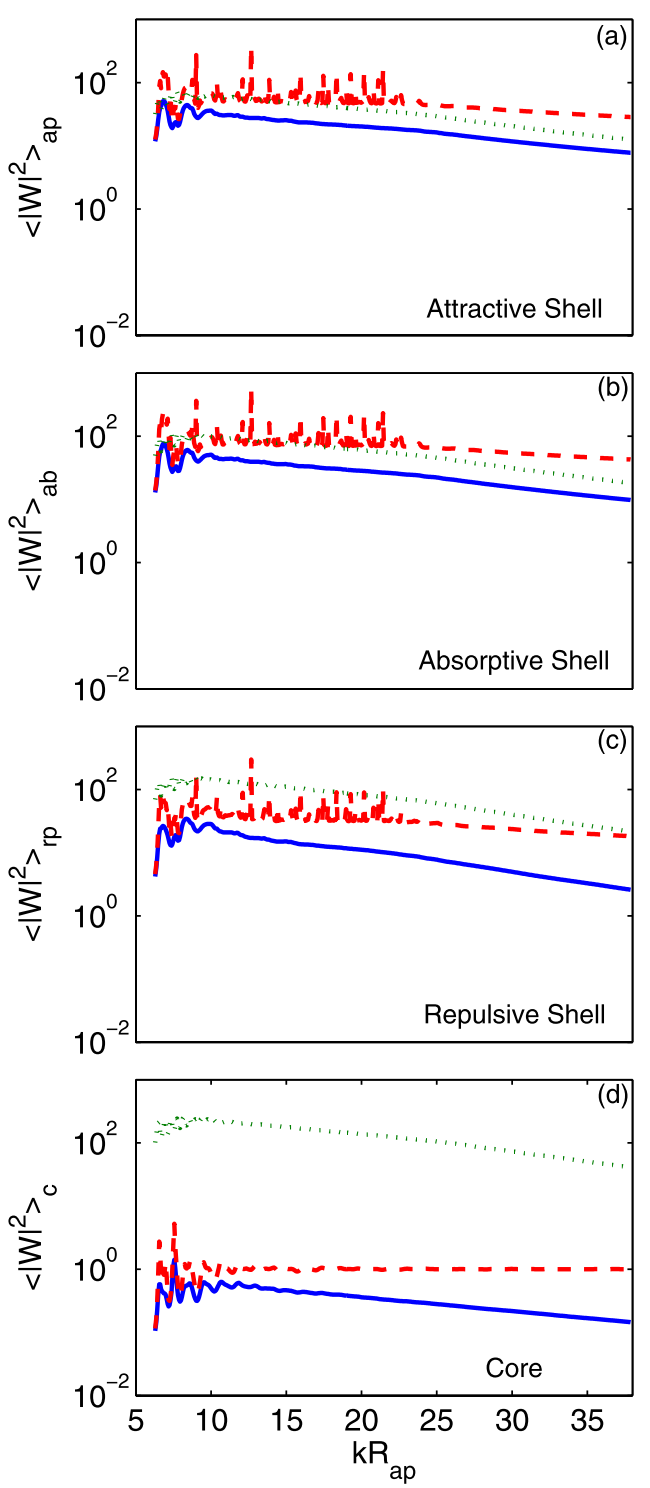

FIG. 8. Vibration average in the attractive potential shell (panel a), the absorptive shell (panel b), the repulsive shell (panel c), and the core (panel c). The lines correspond to the designed device (continuous line), the device without absorption (dashed line), and the device without repulsive potential shell (dotted line).

absorptive lamina on top of the plate. An optimization process has been performed in order to minimize reflections between the different regions defining the device. Moreover, the numerical simulations have been done using an algorithm based on multiple scattering, which has been here developed and described. Since the properties of the materials employed are existent in nature, the proposed device is feasible and, then, an experimental verification is expected in the near future.

The gradient index device has been implemented by exploiting the dependence of the dispersion relation of flexural waves with the plate's thickness. This dependence, which is also found in other types of elastic and electromagnetic open waveguides, could be used to develop similar devices in other systems. For example, devices for vibration isolation in elastic plates immersed in water are one interesting possibility. 


\section{ACKNOWLEDGMENTS}

This work has been supported by the U.S. Office of Naval Research under Grant No. N000140910554.

\section{APPENDIX: SIMULATION METHOD}

The structures studied here are radially inhomogeneous, that is, their parameters depend only on the radial coordinate. The model employed to make numerical simulations is the multilayer scattering method, where the continuous variation of the parameters is discretized into a number $N$ of homogeneous cylindrically symmetric layers. ${ }^{29}$ Figure 9 shows, as an example, the cylindrical structure discretized into $N=10$ layers, each one with different elastic properties. Note that the plate thickness is not a geometrical parameter anymore, but it is introduced in the boundary conditions through the elastic properties of the material. The layers are numbered such that the background corresponds to $n=0$ and the core layer corresponds to $n=N+1$.

The displacement $W_{n}$ in each layer is a solution of Eq. (1) then, in polar coordinates $(r, \theta)$, it can be expanded as

$$
W_{n}(r, \theta)=W_{n}^{(1)}(r, \theta)+W_{n}^{(2)}(r, \theta),
$$

where $W_{n}^{(1)}(r, \theta)$ and $W_{n}^{(2)}(r, \theta)$ are solutions of the Helmholtz and Modified Helmholtz equations, respectively, corresponding to Bessel and Hankel functions (and their modified versions). ${ }^{25}$ Thus, the solution can be explicitly expressed as

$$
\begin{aligned}
W_{n}(r, \theta)= & \sum_{q}\left[A_{n, q}^{(1)} J_{q}\left(k_{n} r\right)+A_{n, q}^{(2)} I_{q}\left(k_{n} r\right)\right] e^{i q \theta} \\
& +\sum_{q}\left[B_{n, q}^{(1)} H_{q}\left(k_{n} r\right)+B_{n, q}^{(2)} K_{q}\left(k_{n} r\right)\right] e^{i q \theta},
\end{aligned}
$$

being $A_{n, q}^{(1)}, A_{n, q}^{(2)}, B_{n, q}^{(1)}, B_{n, q}^{(2)}$ the coefficients of the expansion ( $A$ for the incoming wave towards the center of the cylinder and $B$ for the scattered one) and $k_{n}$ being the wavenumber in the $n$-th layer.

The objective now is to relate the coefficients of each layer with the ones of the previous and next layer. Defining

$$
\hat{A}_{n}=\left[\begin{array}{c}
A_{n, q}^{(1)} \\
A_{n, q}^{(2)}
\end{array}\right], \quad \hat{B}_{n}=\left[\begin{array}{c}
B_{n, q}^{(1)} \\
B_{n, q}^{(2)}
\end{array}\right] .
$$

and from Fig. 9, we can deduce that the relation between the coefficients of layers $n$ and $n-1$ is given by

$$
\begin{gathered}
\hat{A}_{n}=T_{n-1 n} \cdot \hat{A}_{n-1}+R_{n n-1} \cdot \hat{B}_{n}, \\
\hat{B}_{n-1}=R_{n-1 n} \cdot \hat{A}_{n-1}+T_{n n-1} \cdot \hat{B}_{n},
\end{gathered}
$$

where $T_{n-1 n}$ and $R_{n-1 n}$ are the reflection and transmission coefficient matrix (size $2 \times 2$ ) from layer $n-1$ to $n$, respectively. Defining the layer elastic impedance matrices (size $2 \times 2)$ as $\hat{B_{n}}=Z_{n} \cdot \hat{A_{n}}$, the above equations read as

$$
\begin{array}{r}
\hat{A}_{n}=\left(I-R_{n n-1} \cdot Z_{n}\right)^{-1} \cdot T_{n-1 n} \cdot \hat{A}_{n-1}, \\
\hat{A}_{n}=\left(T_{n n-1} \cdot Z_{n}\right)^{-1} \cdot\left(Z_{n-1}-R_{n-1 n}\right) \cdot \hat{A}_{n-1},
\end{array}
$$

from which we can obtain the recursive relation for the coefficient $Z_{n}$ as

$$
\begin{aligned}
& Z_{n-1}=R_{n-1 n}+T_{n n-1} \cdot Z_{n} \cdot X_{n}, \\
& X_{n}=\left(I-R_{n n-1} \cdot Z_{n}\right)^{-1} \cdot T_{n-1 n} .
\end{aligned}
$$

Starting at the last layer $n=N$, since $\hat{B}_{N+1}=0$, the impedance in the last layer $Z_{N}$ is simply

$$
\hat{B}_{N}=R_{N N+1} \cdot \hat{A}_{N} \rightarrow Z_{N}=R_{N N+1} .
$$

The iterative process continues till $n=1$, so all $Z_{n}$ and $X_{n}$ matrix are obtained, then the incoming and scattering coefficients of each layer can be obtained as a function of $\hat{A_{0}}$, which is defined by the external incident field, applying the following relationships:

$$
\begin{gathered}
\hat{B}_{n}=Z_{n} \cdot \hat{A}_{n}, \\
\hat{A}_{n+1}=X_{n} \cdot \hat{A}_{n} .
\end{gathered}
$$

The calculation of the reflection and transmission coefficients of every layer in both propagation directions (to and from the center of the structure), required to realize the aforementioned procedure, is detailed below.

Let us consider a single layer with only one boundary where an incoming wave $\hat{A}_{i}$ impinges on the interface. In Fig. 10(a), the incoming wave $\hat{A}_{n}$ travels towards the center of the cylinder, producing a reflected wave $\hat{B}_{n}$ in the opposite direction and a transmitted one $\hat{A}_{n+1}$ to the next layer. On

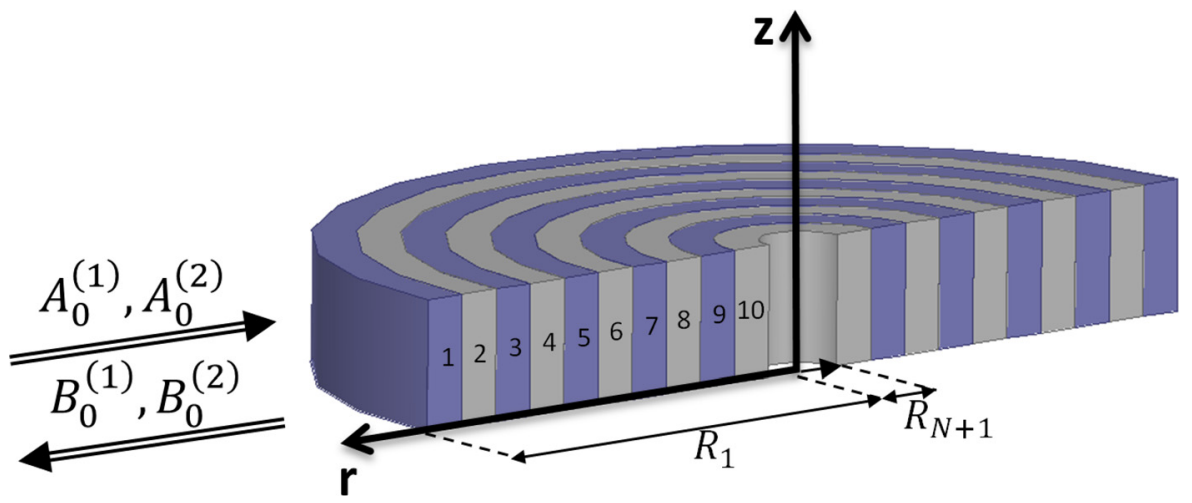

FIG. 9. Multilayered structure employed in the multiple scattering algorithm with $N=10$ layers. The background layer is $n=0$ and corresponds to the region $r>R_{1}$ ) and the core layer is $n=N+1$ and corresponds to the region $r<R_{N+1}$. 


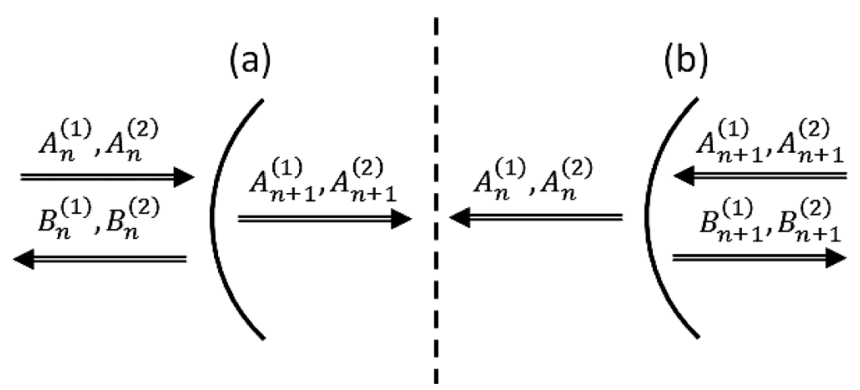

FIG. 10. Mono-layer systems employed to obtain the reflexion and transmission matrices from layer $n$ to $n+1$ (a) and from layer $n+1$ to $n$ (b).

the other hand, in Figure 10(b), the incoming wave $\hat{A}_{n+1}$ travels towards infinity, producing a reflected wave $\hat{B}_{n+1}$ towards the center of the cylinder and a transmitted one $\hat{A}_{n}$ to the previous layer.

To obtain these reflection and transmission matrices, the following boundary conditions ${ }^{23-25,30}$ are imposed:

$$
\left.W_{n}(r, \theta)\right|_{r=R_{n}}=\left.W_{n+1}(r, \theta)\right|_{r=R_{n}},
$$

$$
\begin{gathered}
\left.\frac{\partial W_{n}(r, \theta)}{\partial r}\right|_{r=R_{n}}=\left.\frac{\partial W_{n+1}(r, \theta)}{\partial r}\right|_{r=R_{n}}, \\
\left.M_{r}\left(W_{n}(r, \theta)\right)\right|_{r=R_{n}}=\left.M_{r}\left(W_{n+1}(r, \theta)\right)\right|_{r=R_{n}}, \\
\left.V_{r}\left(W_{n}(r, \theta)\right)\right|_{r=R_{n}}=\left.V_{r}\left(W_{n+1}(r, \theta)\right)\right|_{r=R_{n}},
\end{gathered}
$$

where $M_{r}(f)$ is the radial moment and $V_{r}(f)$ is the KirchoffStress defined as

$$
\begin{gathered}
M_{r}(f)=-D\left[\frac{\partial^{2} f}{\partial r^{2}}+\nu \frac{1}{r} \frac{\partial f}{\partial r}+\nu \frac{1}{r^{2}} \frac{\partial^{2} f}{\partial \theta^{2}}\right], \\
V_{r}(f)=-D \frac{\partial}{\partial r} \Delta f-D(1-\nu) \frac{1}{r^{2}} \frac{\partial}{\partial \theta}\left(\frac{\partial^{2} f}{\partial r \partial \theta}-\frac{1}{r} \frac{\partial f}{\partial \theta}\right) .
\end{gathered}
$$

$D$ being the flexural rigidity and $\nu$ the Poisson ratio. For the first system, after applying these boundary conditions, the coefficients of layers $n$ and $n+1$ are related by ${ }^{30}$

$$
\left[\begin{array}{cccc}
H_{q}\left(\kappa_{n}\right) & K_{q}\left(\kappa_{n}\right) & -J_{q}\left(\kappa_{n+1}\right) & -I_{q}\left(\kappa_{n+1}\right) \\
\kappa_{n} H_{q}^{\prime}\left(\kappa_{n}\right) & \kappa_{n} K_{q}^{\prime}\left(\kappa_{n}\right) & -\kappa_{n+1} J_{q}^{\prime}\left(\kappa_{n+1}\right) & -\kappa_{n+1} I_{q}^{\prime}\left(\kappa_{n+1}\right) \\
S_{n}^{H}\left(\kappa_{n}\right) & S_{n}^{K}\left(\kappa_{n}\right) & -S_{n+1}^{J}\left(\kappa_{n+1}\right) & -S_{n+1}^{I}\left(\kappa_{n+1}\right) \\
T_{n}^{H}\left(\kappa_{n}\right) & T_{n}^{K}\left(\kappa_{n}\right) & -T_{n+1}^{J}\left(\kappa_{n+1}\right) & -T_{n+1}^{I}\left(\kappa_{n+1}\right)
\end{array}\right]\left\{\begin{array}{c}
B_{n, q}^{(1)} \\
B_{n, q}^{(2)} \\
A_{n+1, q}^{(1)} \\
A_{n+1, q}^{(2)}
\end{array}\right\}=(-1)\left[\begin{array}{cc}
J_{q}\left(\kappa_{n}\right) & I_{q}\left(\kappa_{n}\right) \\
\kappa_{n} J_{q}^{\prime}\left(\kappa_{n}\right) & \kappa_{n} I_{q}^{\prime}\left(\kappa_{n}\right) \\
S_{n}^{J}\left(\kappa_{n}\right) & S_{n}^{I}\left(\kappa_{n}\right) \\
T_{n}^{J}\left(\kappa_{n}\right) & T_{n}^{I}\left(\kappa_{n}\right)
\end{array}\right]\left\{\begin{array}{c}
A_{n, q}^{(1)} \\
A_{n, q}^{(2)}
\end{array}\right\},
$$

where

$$
\begin{gathered}
S_{n}^{X}\left(\kappa_{n}\right)=D_{n}\left[\left(q^{2}\left(1-\nu_{n}\right) \mp \kappa_{n}^{2}\right) X_{q}\left(\kappa_{n}\right)-\left(1-\nu_{n}\right) \kappa_{n} X_{q}^{\prime}\left(\kappa_{n}\right)\right], \\
T_{n}^{X}\left(\kappa_{n}\right)=D_{n}\left[\left(q^{2}\left(1-\nu_{n}\right)\right) X_{q}\left(\kappa_{n}\right)-\left(q^{2}\left(1-\nu_{n}\right) \pm \kappa_{n}^{2}\right) \kappa_{n} X_{q}^{\prime}\left(\kappa_{n}\right)\right],
\end{gathered}
$$

and $X=J, I, H, K$; the upper sign is used for $(J, H)$ and the lower sign for $(I, K)$ and $\kappa_{n}=k_{n} R_{n}, k_{n}$ being the wavenumber of the $n$-th layer and $R_{n}$ the radius of the boundary between the layer $n+1$ and $n$.

Similarly, for the second system, the coefficients of layers $n+1$ and $n$ are related by the following system of equations:

$$
\left[\begin{array}{cccc}
J_{q}\left(\kappa_{n+1}\right) & I_{q}\left(\kappa_{n+1}\right) & -H_{q}\left(\kappa_{n}\right) & -K_{q}\left(\kappa_{n}\right) \\
\kappa_{n+1} J_{q}^{\prime}\left(\kappa_{n+1}\right) & \kappa_{n+1} I_{q}^{\prime}\left(\kappa_{n+1}\right) & -\kappa_{n} H_{q}^{\prime}\left(\kappa_{n}\right) & -\kappa_{n} K_{q}^{\prime}\left(\kappa_{n}\right) \\
S_{n+1}^{J}\left(\kappa_{n+1}\right) & S_{n+1}^{I}\left(\kappa_{n+1}\right) & -S_{n}^{H}\left(\kappa_{n}\right) & -S_{n}^{K}\left(\kappa_{n}\right) \\
T_{n+1}^{J}\left(\kappa_{n+1}\right) & T_{n+1}^{I}\left(\kappa_{n+1}\right) & -T_{n}^{H}\left(\kappa_{n}\right) & -T_{n}^{K}\left(\kappa_{n}\right)
\end{array}\right]\left\{\begin{array}{c}
B_{n+1, q}^{(1)} \\
B_{n+1, q}^{(2)} \\
A_{n, q}^{(1)} \\
A_{n, q}^{(2)}
\end{array}\right\}=(-1)\left[\begin{array}{cc}
H_{q}\left(\kappa_{n+1}\right) & K_{q}\left(\kappa_{n+1}\right) \\
\kappa_{n+1} H_{q}^{\prime}\left(\kappa_{n+1}\right) & \kappa_{n+1} K_{q}^{\prime}\left(\kappa_{n+1}\right) \\
S_{n+1}^{H}\left(\kappa_{n+1}\right) & S_{n+1}^{K}\left(\kappa_{n+1}\right) \\
T_{n+1}^{H}\left(\kappa_{n+1}\right) & T_{n+1}^{K}\left(\kappa_{n+1}\right)
\end{array}\right]\left\{\begin{array}{c}
A_{n+1, q}^{(1)} \\
A_{n+1, q}^{(2)}
\end{array}\right\} .
$$

Now, by knowing the definition of the reflection and transmission matrices in the first system

$$
R_{n n+1}=\hat{B}_{n} \cdot\left(\hat{A}_{n}\right)^{-1},
$$

$$
T_{n n+1}=\hat{A}_{n+1} \cdot\left(\hat{A}_{n}\right)^{-1},
$$

and the second system,

$$
R_{n+1 n}=\hat{B}_{n+1} \cdot\left(\hat{A}_{n+1}\right)^{-1},
$$




$$
T_{n+1 n}=\hat{A}_{n} \cdot\left(\hat{A}_{n+1}\right)^{-1} \text {. }
$$

It is straightforward to obtain them from Eqs. (A15) and (A18).

${ }^{1}$ J.-C. Hsu and T.-T. Wu, Phys. Rev. B 74, 144303 (2006).

${ }^{2}$ R. McPhedran, A. Movchan, and N. Movchan, Mech. Mater. 41, 356 (2009).

${ }^{3}$ M. Farhat, S. Guenneau, S. Enoch, A. B. Movchan, and G. G. Petursson, Appl. Phys. Lett. 96, 081909 (2010).

${ }^{4} J$. Pierre, O. Boyko, L. Belliard, J. Vasseur, and B. Bonello, Appl. Phys. Lett. 97, 121919 (2010).

${ }^{5}$ T.-T. Wu, Y.-T. Chen, J.-H. Sun, S.-C. S. Lin, and T. J. Huang, Appl. Phys. Lett. 98, 171911 (2011).

${ }^{6}$ M. Farhat, S. Guenneau, and S. Enoch, EPL 91, 54003 (2010).

${ }^{7}$ M. Oudich, Y. Li, B. M. Assouar, and Z. Hou, New J. Phys. 12, 083049 (2010).

${ }^{8}$ Y. Xiao, J. Wen, and X. Wen, J. Phys. D 45, 195401 (2012).

${ }^{9}$ D. Torrent, D. Mayou, and J. Sanchez-Dehesa, Phys. Rev. B 87, 115143 (2013).

${ }^{10}$ M. Farhat, S. Guenneau, S. Enoch, and A. Movchan, Phys. Rev. B 79, 033102 (2009).

${ }^{11}$ M. Farhat, S. Guenneau, and S. Enoch, Phys. Rev. Lett. 103, 024301 (2009).

${ }^{12}$ N. Stenger, M. Wilhelm, and M. Wegener, Phys. Rev. Lett. 108(1), 014301 (2012).
${ }^{13}$ S. Bramhavar, C. Prada, A. A. Maznev, A. G. Every, T. B. Norris, and T. W. Murray, Phys. Rev. B 83, 014106 (2011).

${ }^{14}$ V. Krylov and F. Tilman, J. Sound Vib. 274, 605 (2004).

${ }^{15}$ V. V. Krylov and R. Winward, J. Sound Vib. 300, 43 (2007).

${ }^{16}$ D. O'Boy, V. V. Krylov, and V. Kralovic, J. Sound Vib. 329, 4672 (2010).

${ }^{17}$ V. Georgiev, J. Cuenca, F. Gautier, L. Simon, and V. Krylov, J. Sound Vib. 330, 2497 (2011).

${ }^{18}$ D. Ross, E. E. Ungar, and E. Kerwin, in Proceedings of Structural Damping, Section 3, edited by J. E. Ruzicka (1959), pp. 49-87.

${ }^{19}$ D. O'Boy and V. V. Krylov, J. Sound Vib. 330, 2220 (2011).

${ }^{20}$ E. Bowyer, D. O’Boy, V. V. Krylov, and J. L. Horner, Appl. Acoust. 73, 514 (2012).

${ }^{21}$ E. Bowyer, D. O’Boy, V. V. Krylov, and F. Gautier, Appl. Acoust. 74, 553 (2013).

${ }^{22} \mathrm{~V}$. Krylov, in Proceedings of the International Conference on Noise and Vibration Engineering (ISMA), edited by P. Sas, D. Moens, and S. Jonckheer (2012), pp. 933-944.

${ }^{23}$ S. Timoshenko, Theory of Plates and Shells (McGraw-Hill, 1940).

${ }^{24}$ K. F. Graff, Wave Motion in Elastic Solids, 2nd ed. (Dover, 1991).

${ }^{25}$ A. W. Leissa, Vibration of Plates, 2nd ed. (NASA, 1969).

${ }^{26}$ E. Narimanov and A. Kildishev, Appl. Phys. Lett. 95, 041106 (2009).

${ }^{27}$ A. Climente, D. Torrent, and J. Sánchez-Dehesa, Appl. Phys. Lett. 100, 144103 (2012).

${ }^{28}$ D. Bies and C. Hansen, Engineering Noise Control: Theory and Practice (Taylor \& Francis, 2009).

${ }^{29}$ L.-W. Cai and J. Sánchez-Dehesa, J. Acoust. Soc. Am. 124, 2715 (2008).

${ }^{30}$ A. Norris and C. Vemula, J. Sound Vib. 181, 115 (1995). 